\title{
SELECCIÓN DE SISTEMAS DE PINTURA PARA CONTROL DE CORROSIÓN ATMOSFÉRICA EN UN CAMPO DE EXPLOTACIÓN PETROLERA EN LA COSTA NORTE DE PERÚ
}

\author{
Claver Hugo Guerra Carvallo ${ }^{*}$
}

\begin{abstract}
RESUMEN
La necesidad de conservar la integridad mecánica de las instalaciones petroleras afectadas por la corrosión atmosférica conduce a estudiar la corrosión atmosférica y categorizarla de acuerdo a estándares existentes en la industria como por ejemplo la norma ISO 9223 "corrosion of metals and alloys - corrosivity of atmospheres - clasification". El campo petrolero, escenario de este estudio, está ubicado en la costa norte de Perú, limita por el oeste con el océano Pacífico, condición geográfica que lo hace susceptible a severa corrosión atmosférica. En este campo se ha conducido un estudio con probetas de acero AISI 1020 que ha permitido caracterizar la corrosión atmosférica en categoría 4 para áreas cercanas al mar y categoría 2 en zonas lejanas. A partir de este estudio la compañía operadora petrolera decidió seleccionar sistemas de pintura eficaces para cada categoría con una durabilidad de 15 años en instalaciones nuevas y 10 años en instalaciones existentes.
\end{abstract}

Palabras clave: Velocidad de corrosión atmosférica, probetas de corrosión, humedad relativa, cloruros en la atmósfera.

\section{SELECTING PAINTING SYSTEMS FOR ATMOSPHERIC CORROSION CONTROL IN AN OIL FIELD LOCATED ON THE NORTH COAST OF PERU}

\begin{abstract}
The need to preserve the mechanical integrity of the oil facilities affected by the atmospheric corrosion leads to study atmospheric corrosion and categorize according to industry standards such as ISO 9223 Corrosion of metals and alloys - corrosivity of atmospheres - clasification. The oil field, scenary of this study, is located on the north coast of Peru, bounded on the west by the Pacific ocean, this geographical condition that makes it susceptible to severe atmospheric corrosion.

An atmospheric corrosion study over steel AISI 1020 specimenes permited characterize the environmental corrosivity category 4 to nearby sea and category 2 in other areas. From this study the operator Oil Company opted for run a field study to select the propers paint systems.

Key words: Atmospheric corrosion rate, corrosion specimens, relative humidity, chlorides in the atmosphere.
\end{abstract}

\footnotetext{
$a^{*}$ Licenciado en Química, Universidad Nacional Mayor de San Marcos, Facultad de Química. Av.Universitaria s/n.Lima. claverguerra@yahoo.es
} 


\section{INTRODUCCIÓN}

La corrosión atmosférica en el campo petrolero ubicado en la costa norte de Perú, motivo del estudio, ha sido responsable de gastos operativos anuales de aproximadamente 1 millón de dólares. La zona en la cual las instalaciones metálicas son más afectadas por la corrosión atmosférica es la zona cercana al mar.

En el periodo de septiembre del 2010 a septiembre del 2011 fue conducido un estudio dirigido a categorizar la velocidad de corrosión atmosférica. El estudio consideró determinar la velocidad de corrosión de acuerdo a la norma ISO 9223-12 ${ }^{1}$ y para ello se usó probetas construidas de acero ISO-SAE 1020 de $100 \mathrm{~mm}$ de ancho, $150 \mathrm{~mm}$ de largo y $1 \mathrm{~mm}$ de espesor. La velocidad de corrosión fue determinada por pérdida de peso en un año de exposición.

El estudio concluyó que la corrosión atmosférica podía categorizarse en categoría 4, corrosión severa, para las zonas cercanas al mar, siendo la ecuación de velocidad de corrosión correspondiente en $\mu \mathrm{m} /$ año igual a $414.95 \mathrm{t}^{-0.3206}$, donde $\mathrm{t}$ se expresa en días; y categoría 2, corrosión baja, en áreas lejanas, correspondiéndole una ecuación de velocidad de corrosión en $\mu \mathrm{m} /$ año igual a $161.33 \mathrm{t}^{-0.3729}$, donde t se expresa en días. Ver figuras 1 y 2.

Basados en los resultados de este estudio se emprendió un proyecto para seleccionar los sistemas de pintura que garanticen la integridad mecánica de las instalaciones metálicas de los efectos de la corrosión atmosférica. Este proyecto se inició en abril del 2011 y los resultados aquí mostrados corresponden hasta junio del 2013.

A continuación se muestra los gráficos de velocidad de corrosión atmosférica sobre probetas construidas con acero AISI - SAE 1020 en los ambientes atmosféricos del presente estudio.

\section{Velocidad de corrosión en función del tiempo de exposición. \\ Estación ubicada a $820 \mathrm{~m}$ del mar}

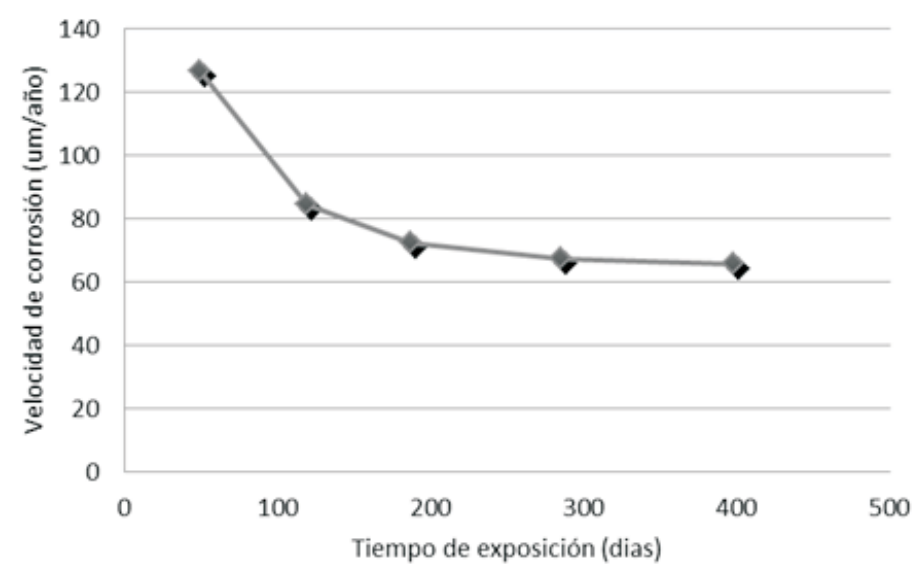

Figura 1. Velocidad de corrosión sobre probetas desnudas de acero AISI - SAE 1020, expuesta durante un año en una estación cercana al mar. Ambiente C4. 


\section{Velocidad de corrosión en función del tiempo de exposición. \\ Estación ubicada a $5900 \mathrm{~m}$ del mar}

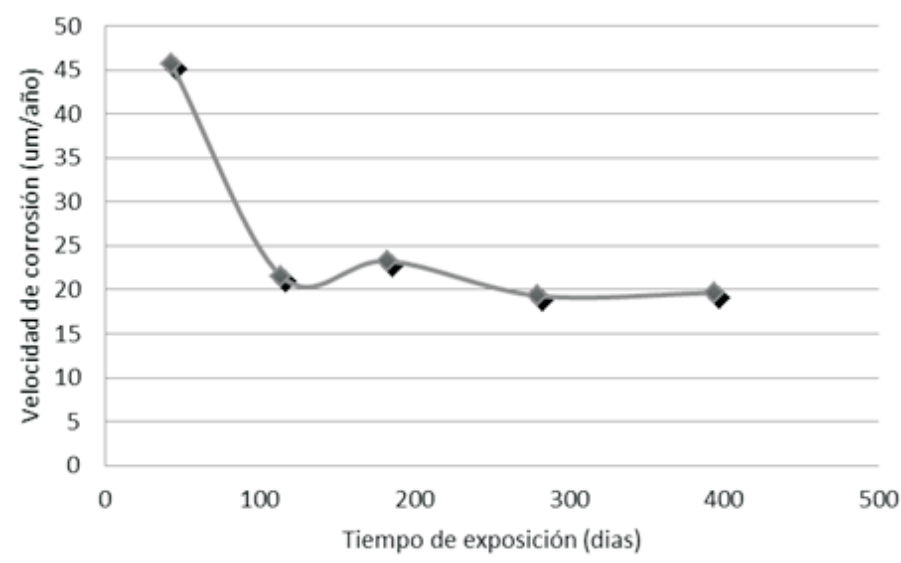

Figura 2. Velocidad de corrosión sobre probetas desnudas de acero AISI SAE 1020, expuesta durante un año en una estación lejana al mar. Ambiente C2.

El protocolo del proceso de selección de sistemas de pintura ha sido diseñado teniendo en consideración la norma ISO 12944 - 07 Paints and varnishes - Corrosion protection of steel structures by protective paint systems - Part 5: Protective paint systems ${ }^{2}$.

En el presente estudio hemos utilizado los siguientes sistemas de pintura:

Ambiente categoría de corrosión 4

- $\quad$ Primera capa.- Epóxido Zn poliamida, de bajo contenido de componentes orgánicos volátiles y pigmentos de $\mathrm{Zn}$.

- Segunda capa.- Epóxido - poliamina

- Tercera capa.- Poliuretano alifático acrílico.

Ambiente categoría de corrosión 2

- $\quad$ Primera capa.- Epóxido - poliamida, Epóxido - poliamina

- Segunda capa.- Epóxido - poliamida

- Tercera capa.- Poliuretano alifático acrílico, Epóxido.

Estos sistemas de pintura han sido aplicados en probetas de acero ASTM A36. Antes de aplicar la primera capa de pintura la superficie de la probeta fue limpiada hasta apreciarse el material libre de óxido, grasa y todo tipo de suciedad ${ }^{2}$.

Las características físicoquímicas y de aplicación de estos tipos de pinturas son los siguientes: 


\section{Epóxido poliamida y Epóxido poliamina}

Una resina epóxido o poli epóxido es un polímero termoestable que se endurece cuando se mezcla con un agente catalizador o «endurecedor». Las resinas epóxidos más frecuentes son producto de una reacción entre epiclorohidrina y bisfenol A; su fórmula molecular es la siguiente.<smiles>CC(C)(C)c1ccc(OCC(O)COc2ccc(C(C)(C)c3ccc(OCC4CO4)cc3)cc2)cc1</smiles>

Las combinaciones más frecuentes, en el área de productos para mantenimiento industrial, son resinas epóxidos con poliamida y poliamina.

Las resinas epóxidos reaccionan químicamente con estas sustancias, dando como resultado revestimientos termoestables, también conocidos como convertibles. Estos polímeros tienen diversas propiedades que los caracterizan, tales como: resistencia a los solventes y a algunos ácidos, álcalis y sales.

Películas basadas en resinas epóxidos combinadas con poliamidas o poliaminas adecuadamente pigmentadas, ofrecen una notable adherencia sobre diferentes substratos formando una película dura, resistente a la abrasión y de alto desempeño por sus propiedades físicas y químicas. Las características más importantes son: gran resistencia a ambientes químicamente agresivos y durabilidad en trabajos pesados que exigen resistencia a los daños mecánicos.

Las combinaciones de resinas epóxido con poliamida resultan en productos de excelente resistencia al agua y a la humedad.

Las resinas epóxido combinadas con poliamina ofrecen un amplio abanico de opciones para la protección de agentes químicos de alta agresividad.

\section{Resinas epóxido enriquecidas con $\mathbf{Z n}$}

La aplicación de pinturas enriquecidas con $\mathrm{Zn}$ es eficiente en el control de la corrosión en ambientes marinos y de severa corrosión atmosférica. Es común aplicarlas como una primera capa para conseguir un largo tiempo de vida de protección. La adición de partículas de Zn tiene el propósito de proveer protección catódica al sustrato de acero, por consiguiente se espera que en un largo tiempo el sustrato se proteja por la formación de una capa de productos de corrosión que refuerza el efecto barrera de la pintura.

El contenido y tamaño de las partículas de Zn así como el material aglutinante impacta notablemente en la eficacia de este tipo de pinturas ${ }^{3}$. Las partículas de Zn, esférico o lamelar, es dispersado en un aglutinante inorgánico, orto silicatos, u orgánico, usualmente epóxido. Estas partículas deben estar en contacto eléctrico entre ellas y el sustrato, para asegurar una buena conducción eléctrica dentro del recubrimiento. Bajo estas condiciones se establece un acoplamiento galvánico entre el Zn y el sustrato, el cual es noble con respecto al Zn. El Zn se disuelve actuando como un pigmento de sacrificio. 
Los potenciales de oxidación se muestran a continuación ${ }^{4}$
$\mathrm{Zn}\left\langle-------->\mathrm{Zn}^{+2}+2 \mathrm{e}-\right.$
Potencial de oxidación $-0,76$ voltios
$\mathrm{Fe}<-------->\mathrm{Fe}^{+2}+2 \mathrm{e}-$
Potencial de oxidación $\quad-0,44$ voltios

Para asegurar un buen contacto eléctrico con el sustrato, usualmente se utiliza Zn en más de $60 \%$ en volumen. El tamaño de estos pigmentos cuando son esféricos es de 5 a 10 micras de diámetro.

\section{Poliuretanos}

El poliuretano (PU) es un polímero que se obtiene mediante condensación de bases hidroxílicas combinadas con isocianatos. Su grupo funcional es el siguiente.

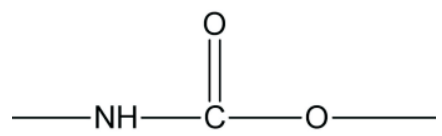

Los revestimientos poliuretánicos, de modo general, se presentan como pinturas de dos componentes. La base puede ser un poliéster y el catalizador un poli - isocianato. Una vez mezclados, estos componentes reaccionan para formar un polímero que da como resultado una película que conjuga características estéticas, físicas y químicas distintas a los demás revestimientos de secado al aire. Entre los diversos tipos de poliuretanos comercializados en la actualidad, citaremos a dos grupos principales que se diferencian por características bien marcadas: Los poliuretanos aromáticos usados como primera mano, manos intermedias o terminaciones, son sensibles a la radiación ultravioleta, pues cuando son expuestos a la acción de la intemperie se tornan amarillentos y con pérdida de brillo por la acción del sol. Estos poliuretanos aromáticos son recomendados para el pintado de superficies que no estén expuestas a la acción de la intemperie. Los poliuretanos alifáticos se caracterizan por la excelente resistencia a la intemperie, bajo las condiciones atmosféricas más diversas, tales como: áreas marítimas, áreas con gran incidencia de la radiación solar, áreas industriales altamente contaminadas y húmedas, áreas donde la deposición de residuos en suspensión sobre la superficie pintada es de forma agresiva para el revestimiento. Estos factores adversos no producen cambios en la protección anticorrosiva, ni el deterioro visual en cuanto a brillo o retención de color, excepto a muy largo plazo. Para especificar un esquema de pintura en áreas agresivas se debe considerar no solamente el costo inicial del trabajo de pintado, por la simple evaluación del precio de adquisición de las pinturas, sino tener en cuenta la economía basada en la vida útil de las superficies pintadas en función de los años de servicio estimados.

Con la selección de un esquema formado por un primer anticorrosivo de base epoxi, combinado con una terminación a base de un poliuretano, este objetivo será plenamente atendido por su duradera función protectora.

\section{PARTE EXPERIMENTAL}

El trabajo experimental fue conceptualizado para obtener los siguientes logros

- El sistema de pintura debe ofrecer importante mitigación de la corrosión atmosférica.

- El sistema de pintura debe ser factible de aplicarlo con técnicas de pintado existentes en el mercado local. 
- El sistema de pintura debe mantener su integridad en un periodo de tiempo que garantice continuidad normal de la actividad productiva. Establecimos este periodo en 15 años para instalaciones nuevas.

La técnica de selección del sistema de pintura estuvo basada en el uso de probetas de acero ASTM A36, limpiadas inicialmente hasta metal desnudo y a continuación pintadas, cada capa a diferente espesor y diferente acabado. Cabe señalar que en toda etapa de este proceso de selección participaron representantes técnicos de cuatro compañías proveedoras de pintura de amplio prestigio nacional. Los sistemas de pintura fueron propuestos y aplicados por los proveedores de pintura . La pintura se aplicó sobre probetas pulidas hasta metal desnudo de acuerdo a la Norma ISO $12944-07^{2}$. Las dimensiones de las probetas fueron $100 \mathrm{~mm}$ de ancho, $150 \mathrm{~mm}$ de largo y $3 \mathrm{~mm}$ de espesor. Las probetas fueron instaladas en dos estaciones: una estación cercana al mar (ambiente categorizado C4) y la otra estación ubicada en ambiente $\mathrm{C} 2$.

Las variables evaluadas fueron las siguientes:

\section{Grado de oxidación ${ }^{5}$.}

- La norma correspondiente está basada en la designación del grado de herrumbre u oxidación que presenta un sistema de recubrimientos y se obtiene por comparación con una serie de dibujos que relacionan el grado de oxidación con el porcentaje de área afectada.

- Cumplido el período de exposición, inspeccionamos cada probeta para observar si existe la presencia de corrosión sobre la superficie expuesta.

La categorización del impacto es calificado de acuerdo con los indicadores de la tabla 1.

Tabla 1. Tabla de categorización del grado de oxidación ${ }^{5}$.

\begin{tabular}{cc}
\hline $\begin{array}{c}\text { Grado de } \\
\text { oxidación }\end{array}$ & $\begin{array}{c}\text { Porcentaje de área afectada } \\
10\end{array}$ \\
9 & Sin oxidación o menos de $0,01 \%$ de área herrumbrada. \\
& Menos de $0,03 \%$ de área herrumbrada. \\
8 & Pocas manchas de herrumbre aisladas, menos de $0,1 \%$ de área \\
7 & herrumbrada. \\
6 & Menos de $0,3 \%$ de área herrumbrada. \\
5 & Manchas generalizadas de herrumbre, pero menos de $1 \%$ de área \\
4 & herrumbrada. \\
3 & Aproximadamente una sexta parte del área herrumbrada. \\
2 & Aproximadamente una tercera parte del área herrumbrada. \\
1 & Aproximadamente la mitad del área herrumbrada. \\
0 & Aproximadamente $100 \%$ del área herrumbrada.
\end{tabular}




\section{Grado de ampollamiento ${ }^{6}$}

- La norma correspondiente está basada en la designación del grado de ampollamiento que presenta un sistema de recubrimientos, la cual se obtiene por comparación con una serie de dibujos que relacionan el grado de ampollamiento según el tamaño de la ampolla (2, 4, 6,8 ) y la densidad de las mismas F (poca), M (medio densa), D (densa) y VD (muy densa) (tabla 2).

Tabla 2. Categorización por el tamaño y densidad de ampollamiento ${ }^{6}$

\begin{tabular}{|c|c|c|c|}
\hline Número & Tamaño & Grado & Densidad \\
\hline 10 & 0 & 0 & $\begin{array}{c}\text { Sin } \\
\text { ampollamiento }\end{array}$ \\
\hline 8 & $0-1 \mathrm{~mm}$ & $\mathrm{~F}$ & Poca \\
\hline 6 & $1-2 \mathrm{~mm}$ & $\mathrm{M}$ & Media \\
\hline 4 & $2-3 \mathrm{~mm}$ & MD & Medio densa \\
\hline 2 & $3-5 \mathrm{~mm}$ & D & Densa \\
\hline 0 & $+5 \mathrm{~mm}$ & VD & Muy densa \\
\hline
\end{tabular}

\section{Grado de deterioro en la zona de incisión ${ }^{7}$}

- La norma se basa en la designación del grado de daño producido en la zona de incisión. Al inicio de la exposición se efectúa una incisión en la película de pintura con una navaja. La profundidad de la incisión debe llegar hasta tocar el metal o sustrato (tabla 3).

Tabla 3. Tabla de categorización del daño en la zona de incisión ${ }^{7}$.

\begin{tabular}{cc}
\hline Milímetros & Grado \\
Cero & 10 \\
0 a 0,5 & 9 \\
0,5 a 1 & 8 \\
1 a 2 & 7 \\
2 a 3 & 6 \\
3 a 5 & 5 \\
5 a 7 & 4 \\
7 a 10 & 3 \\
10 a 13 & 2 \\
13 a 16 & 1 \\
16 & 0 \\
\hline
\end{tabular}

Otras variables de control sobre las probetas pintadas fueron las siguientes:

- Tizado. Para evaluar deterioro por radiación UV.

- Espesor de pintura. Medición de pérdida de pintura por el ambiente.

- Depósitos de hongos. Observar capacidad de favorecer el crecimiento de microrganismos.

- Contenido de cloruros en la atmósfera, humedad relativa ${ }^{8}$, velocidad del viento, temperatura ambiental (tabla 4). 
Tabla 4. Variables ambientales

\begin{tabular}{lcc}
\hline \multicolumn{1}{c}{ Estación } & Ambiente & Ambiente \\
& $\mathrm{C} 4$ & $\mathrm{C} 2$ \\
Altitud, m & 41 & 295 \\
Velocidad del viento promedio, millas por hora & 9.5 & 10 \\
Temperatura mínima, ${ }^{\circ} \mathrm{C}$ & 34 & 25 \\
Temperatura máxima, ${ }^{\circ} \mathrm{C}$ & 32 & 36 \\
Humedad relativa promedio, \% & 76 & 58 \\
Cloruros en la atmósfera promedio, $\mathrm{mg} / \mathrm{m} 2$. día, & 109.2 & 92.6 \\
\hline
\end{tabular}

\section{RESULTADOS Y DISCUSIÓN}

Los resultados al cabo de dos años de exposición se exhiben en la tabla 5.

Tabla 5. Resultados de evaluación en dos años de exposición. El poliuretano utilizado en la capa externa del sistema de pintura fue poliuretano alifático.

\begin{tabular}{|c|c|c|c|c|c|c|c|c|c|}
\hline & $\begin{array}{l}\text { ASTM } \\
\text { D610 }\end{array}$ & $\begin{array}{l}\text { ASTM } \\
\text { D714 }\end{array}$ & $\begin{array}{l}\text { ASTM } \\
1654\end{array}$ & $\begin{array}{l}\text { Pérdida } \\
\text { de brillo }\end{array}$ & $\begin{array}{l}\text { Pérdida } \\
\text { de color }\end{array}$ & Fiscalización & Tizado & Erosión & Hongos \\
\hline \multicolumn{10}{|c|}{ Ambiente $\mathrm{C} 4$} \\
\hline $\begin{array}{l}\text { Zn orgánico, } \\
\text { Epóxido } \\
\text { Poliuretano }\end{array}$ & 10 & 10 & 10 & No & No & No & No & No & No \\
\hline $\begin{array}{l}\text { Zn inorgánico, } \\
\text { Epóxido, } \\
\text { Poliuretano }\end{array}$ & 10 & 10 & 10 & No & No & No & No & No & No \\
\hline $\begin{array}{l}\text { Epóxido, } \\
\text { Poliuretano }\end{array}$ & 10 & 10 & 10 & No & No & No & No & No & No \\
\hline \multicolumn{10}{|c|}{ Ambiente $\mathrm{C} 2$} \\
\hline $\begin{array}{l}\text { Epóxido, } \\
\text { Poliuretano }\end{array}$ & 10 & 10 & 10 & No & No & No & No & No & No \\
\hline $\begin{array}{l}\text { Epóxido, } \\
\text { Poliuretano }\end{array}$ & 10 & 10 & 10 & No & No & No & No & No & No \\
\hline
\end{tabular}

Los sistemas de pintura con capa exterior tipo epóxido expuesta por 85 días, aun en ambiente C2, pierde brillo, color y muestra tizado, por esta razón las probetas correspondientes fueron retiradas cumplido el periodo de exposición de 85 días.

Se denomina tizado a la cualidad de la pintura de formar un polvo debido a su reacción con el medio ambiente, especialmente con la radiación UV. Al pasar el dedo sobre estas pinturas el polvo se adhiere como una tiza.

Todas las probetas estudiadas durante dos años de exposición en ambiente C4 y C2 no mostraron deterioro en la zona de incisión.

Ninguna de las probetas ensayadas durante dos años de exposición mostró ampollamiento 


\section{CONCLUSIONES}

- Los poliuretanos alifáticos muestran alta resistencia a la radiación UV, mantienen su brillo durante los dos años de exposición.

- Los epóxidos no resisten a la radiación UV, en menos de dos meses pierden brillo y se tizan.

- Para ambiente C4 concluimos que el siguiente sistema de pintura es muy efectivo:

Primera capa: Epóxido con pigmentos de Zn

Segunda capa: Epóxido

Tercera capa: Poliuretano alifático

- Para ambiente C2 concluimos que el siguiente sistema de pintura es muy efectivo:

Primera capa: Epóxido

Segunda capa: Epóxido

Tercera capa: Poliuretano.

\section{RECOMENDACIONES}

- Se recomienda como paso previo a la selección de un sistema de pintura realizar un estudio de caracterización y categorización de la corrosión atmosférica con el propósito de acotar el número de sistemas de pintura que serán estudiadas.

- El alto costo de un proceso de pintado y la necesidad de conservar la integridad mecánica de las instalaciones metálicas, debido a fenómenos de corrosión atmosférica, exige realizar un sistemático estudio de selección de sistemas de pintura en campo. Si bien la selección de sistemas suele efectuarse mediante pruebas de laboratorio, hay muchos factores ambientales que influyen en la corrosión atmosférica y estos en su totalidad no pueden reproducirse en un ensayo de laboratorio; por ejemplo, el impacto de la presencia de hongos, cambios de temperatura por lluvias, cambios súbitos de humedad, etc.

\section{BIBLIOGRAFÍA}

1. ISO 9223 - 12, British standard, Corrosion of Metals and alloys - corrosivity of atmospheres - clasification, UK, 2012

2. ISO 12944 - 5 - 07, British standard, Paints and varnishes - Corrosion protection of steel structures by protective paint systems - Part 5: Protective paint systems, UK, 2007.

3. Nadia Hammouda, Hacène Chadli, Gildas Guillemot, Kamel Belmokre, The Corrosion Protection Behaviour of Zinc Rich Epoxy Paint in 3\% $\mathrm{NaCl}$ Solution, Laboratoire d'Anticorrosion Matériaux Environnement, Université du 20 Août 1955, Skikda, Algérie. Laboratoire de Métallurgie et Génie des Matériaux, Université de Badji Mokhtar, Annaba, Algérie Laboratoire de Métallurgie Physique et Génie des Matériaux, Ecole Nationale Supérieure d'Arts et Métiers de Lille, Lille Cedex, France? E-mail: hammoudanad@yahoo.fr? January 16, 2011; revised March 16, 2011; accepted March 25, 2011. Advances in Chemical Engineering and Science, 2011, 1, 51-60 doi:10.4236/aces.2011.12009 Published Online April 2011 (http://www.scirp.org/journals/aces).

4. J.C.Scully, Fundamentos de la Corrosion, Editorial Alhambra S.A. Mexico, 1968.

5. ASTM D 610-08, ASTM international, Standard Test Method for Evaluating Degree of Rusting on Painted Surfaces, USA, 2008 
6. ASTM D 714-09, ASTM international, Standard Test Method for Evaluating Degree of Blistering of Paints, USA. 2009.

7. ASTM D 1654-08, ASTM international, Standard Test Method for Evaluating Degree of Painted or Coated Speciment Subjected to corrive Envioronments, USA, 2008.

8. ASTM E 337 - 07, ASTM international, Método estándar para la medición de Humedad con un Psicrómetro (medición de temperaturas de bulbo seco y bulbo húmedo, USA, 2007. 